\title{
The intestinal microbiome of fish under starvation
}

\author{
Jun Hong Xia ${ }^{1}$, Grace Lin ${ }^{1}$, Gui Hong Fu', Zi Yi Wan ${ }^{1}$, May Lee ${ }^{1}$, Le Wang ${ }^{1}$, Xiao Jun Liu ${ }^{1}$ and Gen Hua Yue ${ }^{1,2^{*}}$
}

\begin{abstract}
Background: Starvation not only affects the nutritional and health status of the animals, but also the microbial composition in the host's intestine. Next-generation sequencing provides a unique opportunity to explore gut microbial communities and their interactions with hosts. However, studies on gut microbiomes have been conducted predominantly in humans and land animals. Not much is known on gut microbiomes of aquatic animals and their changes under changing environmental conditions. To address this shortcoming, we determined the microbial gene catalogue, and investigated changes in the microbial composition and host-microbe interactions in the intestine of Asian seabass in response to starvation.

Results: We found 33 phyla, 66 classes, 130 orders and 278 families in the intestinal microbiome. Proteobacteria (48.8\%), Firmicutes (15.3\%) and Bacteroidetes (8.2\%) were the three most abundant bacteria taxa. Comparative analyses of the microbiome revealed shifts in bacteria communities, with dramatic enrichment of Bacteroidetes, but significant depletion of Betaproteobacteria in starved intestines. In addition, significant differences in clusters of orthologous groups (COG) functional categories and orthologous groups were observed. Genes related to antibiotic activity in the microbiome were significantly enriched in response to starvation, and host genes related to the immune response were generally up-regulated.

Conclusions: This study provides the first insights into the fish intestinal microbiome and its changes under starvation. Further detailed study on interactions between intestinal microbiomes and hosts under dynamic conditions will shed new light on how the hosts and microbes respond to the changing environment.
\end{abstract}

Keywords: Fish, Microbiome, Starvation, Stress, Interaction, Nutrition

\section{Background}

Wildlife species can often be affected by starvation due to changes in environmental factors (e.g., temperatures, salinity and oxygen concentration). Starvation not only affects the nutritional and health status of animals, but also the microbes in their intestines [1]. Physiological changes during starvation drives the animals and their intestinal microbes to rapidly acclimate to the situation [1-3].

The composition and interactions of the gut microbiota may affect the amount of energy extracted from the diet and energy harvest [4,5], and play an important role in the metabolism of dietary substrates and immune system modulation [6]. The balance of gut microbial community composition can be altered by many factors,

\footnotetext{
* Correspondence: genhua@tll.org.sg

${ }^{1}$ Molecular Population Genetics Group, Temasek Life Sciences Laboratory, 1 Research Link, National University of Singapore, Singapore 117604, Republic of Singapore

${ }^{2}$ Department of Biological Sciences, National University of Singapore, 14 Science Drive 4, Singapore 117543, Singapore
}

including stress $[7,8]$, antibiotic exposure [9], nutritional status [10], age [11], degree of hygiene [12] and bacterial infection [13]. Diets play a dominant role in shaping gut microbiota and altering the metabolism and population sizes of key symbiont species, resulting in biological changes to the host [14]. An altered microbiota in the intestine can lead to altered immune functions of hosts, and also increase the risk of disease [14,15]. However, as studies on gut microbiomes have largely been performed on humans and land animals [16-21], not much is known on gut microbiomes and their changes under changing environmental conditions in organisms living in aquatic habitats.

Studies of bacteria community are traditionally carried out on the basis of representative genomes and signature genes such as $16 \mathrm{~S}$ ribosomal RNA (rRNA). However, analyses of $16 \mathrm{~S}$ rRNA can only appraise the phylogenetic composition of a sample and provide no direct information about its functional capabilities [22]. Full scale metagenomics can augment the information content of the data

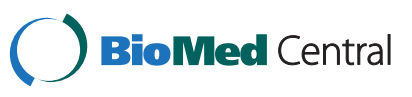

(C) 2014 Xia et al.; licensee BioMed Central Ltd. This is an Open Access article distributed under the terms of the Creative Commons Attribution License (http://creativecommons.org/licenses/by/2.0), which permits unrestricted use, distribution, and reproduction in any medium, provided the original work is properly credited. 
generated by not only determining the relative abundance of all genes, but a description of the functional potential of each community as well. Compared with the signature gene-based methods, this new technique gives a much broader description than phylogenetic surveys [23]. Recently, an analysis of $16 \mathrm{~S}$ rRNA was performed to assess the bacteria composition in the grass carp [24,25] and the zebrafish [26]. However, little is known about the gene content of fish microbiota and the effects of starvation on microbial populations in fish.

The Asian seabass Lates calcarifer is an important farmed foodfish species. The fish has the ability to tolerate all levels of salinity from fresh to seawater allowing them to be cultured in both environments. This species feeds on crustaceans, mollusks, and smaller fish [27]. In this study, we sequenced the metagenomic DNA isolated from the intestine of the Asian seabass using a Hiseq 2000 sequencer. We characterized the intestinal microbiome, and analyzed the influence of starvation on the composition of the gut microbial communities. By using comparative metagenomic studies and analyzing expression of selected host genes with quantitative reverse-transcriptase PCR (qRT-PCR), we have constructed a primary microbial gene catalogue, and investigated the changes of the microbial composition and the host-microbe interactions in the intestine under starvation. Our analysis suggests variable microbiomes and host-microbiota signatures in the fish intestines in response to starvation.

\section{Results and discussion}

\section{Metagenomic analysis suggests variable microbiomes in} fed vs. starved seabass intestines

The intestine is one of the major organs in fish that interacts with the environment, and is involved in adaptations and stress responses. The intestinal microbiota are composed of a diverse and vast population of microorganisms [28]. To characterize and compare the microbial communities in the Asian seabass intestine in response to starvation, we sequenced two metagenomic DNA samples isolated from Asian seabass intestines at eight days post fasting challenge (experimental sample) and from fed seabass intestines (control) by using the Illumina Hiseq 2000 sequencing system. The sequencing yielded 71,254,936 reads for the experimental sample and 64,649,316 reads for the control sample. The sequences gave an overall average length of $101 \mathrm{bp}$ and represented $13.7 \mathrm{~Gb}$ of DNA data. After trimming of the low-quality sequences and adaptors, 69,893,230 and 62,408,866 high-quality reads for the experimental sample and the control sample were obtained. The high quality reads were classified from phylum to family using the program MetaPhlAn [29] with the default settings.

In the control sample, we found that $96.3 \%$ of the metagenomes were assigned to bacteria and $3.7 \%$ were assigned to Archaea. The metagenome included 33 phyla, 66 classes, 130 orders and 278 families (Additional file 1). Proteobacteria (48.8\%), Firmicutes (15.3\%), Bacteroidetes (8.2\%) and Fusobacteria (7.3\%) were the four most abundant bacteria phyla (Figure 1). The results were generally consistent with those observed in the intestinal samples of other fish species. In grass carp, Proteobacteria and Firmicutes were dominant in the gut bacteria [25], and in adult zebrafish, Proteobacteria (79.4\%) and Fusobacteria (13.6\%) phyla were common members of the intestinal microbiota [26]. However, there are some differences in the intestinal microbiota among different fish species. For example, in grass carp, Actinobacteria (more than 10\%) were the most prevalent members of the intestinal bacterial communities, and they were more abundant than Bacteriodetes [25]. In our study, only $1.1 \%$ and $0.5 \%$ of the microbiota members were Actinobacteria in the control sample and in the experimental sample, respectively. Therefore, Actinobacteria were much less abundant than Bacteriodetes (8.2\%) in the Asian seabass intestine. Diet plays a dominant role in shaping gut microbiota and changing key populations $[14,30,31]$. The grass carp is an herbivorous species, while Asian seabass is a strict carnivore, feeding on crustaceans, mollusks and smaller fish in the wild. Therefore, the difference in bacterial communities between grass carp and Asian seabass may be caused by evolved differences that have arisen in the context of differing diets. In addition, our data supported prior findings suggesting that gut microbiota differ between fish and mammalian intestines. Firmicutes and Bacteroidetes were the most dominant phyla in mammals [32,33], whereas Proteobacteria were the most abundant bacteria phyla in fish. This difference suggests that gut environments differ in their selectivity/hospitability for bacterial proliferation.

In the experimental sample, we found that $97.7 \%$ of the metagenomes were assigned to bacteria, and $2.3 \%$ were assigned to Archaea (Additional file 1). Proteobacteria (39.1\%), Bacteroidetes (36.0\%) and Firmicutes (10.1\%) were the three most abundant bacterial species in the experimental sample (Figure 1). Both metagenomes showed very small differences in diversity when reviewed in light of the numbers of present taxa (Additional file 1). However, dramatic differences in microbial community composition of the fish intestine across samples were observed (Figure 1 and Table 1). We found that a total of three orders and four families showed apparent shifts in relative abundance in response to starvation. More detailed information on these shifts in microbial community composition was presented in Additional file 1. These findings are consistent with previous studies in humans, in which the relative abundances of different bacterial species in the gut microbiota were highly sensitive to diet $[10,32]$.

Significantly more reads were assigned to the Bacteroidetes phylum in the experimental sample (36\%) as compared to 


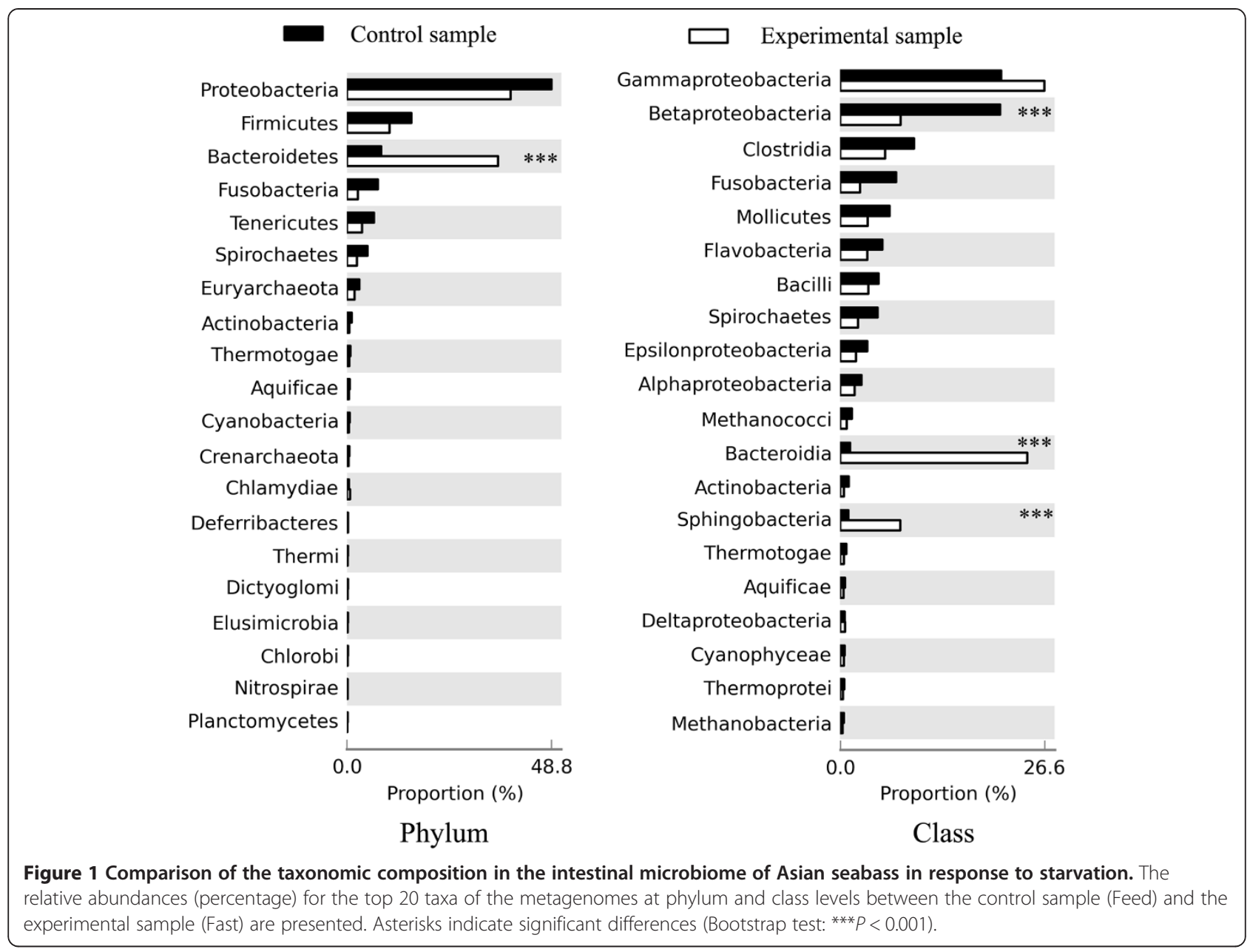

Table 1 Shift in intestinal microbiota in Asian seabass in response to starvation

\begin{tabular}{lllll}
\hline Rank & Classification & Control (\%) & Experimental (\%) & $\boldsymbol{P}$-value \\
\hline Phylum & Bacteroidetes & 8.2 & 36.0 & $<1 \mathrm{E}-15$ \\
Class & Betaproteobacteria & 20.8 & 7.9 & $<1 \mathrm{E}-15$ \\
Class & Bacteroidia & 1.3 & 24.4 & $<1 \mathrm{E}-15$ \\
Class & Sphingobacteria & 1.1 & 7.8 & $<1 \mathrm{E}-15$ \\
Order & Burkholderiales & 20.3 & 7.4 & $<1 \mathrm{E}-15$ \\
Order & Bacteroidales & 1.3 & 24.4 & $<1 \mathrm{E}-15$ \\
Order & Sphingobacteriales & 1.1 & 7.8 & $<1 \mathrm{E}-15$ \\
Family & Oxalobacteraceae & 20.0 & 7.1 & $<1 \mathrm{E}-15$ \\
Family & Sphingobacteriaceae & 1.1 & 7.8 & $<1 \mathrm{E}-15$ \\
Family & Bacteroidaceae & 0.6 & 23.2 & $<1 \mathrm{E}-15$ \\
Family & Aeromonadaceae & 0.1 & 2.4 & $<1 \mathrm{E}-15$ \\
\hline
\end{tabular}

The significant changes in microbial composition (relative abundance, \%) from family to phylum in the fish intestines in response to starvation are presented. $P$-value indicates significance level as calculated using a bootstrap statistical test. the control sample (8.2\%). At the class level, Bacteroidia (1.3\% in the control sample vs. $24.4 \%$ in the experimental sample) and Sphingobacteria (1.1\% in the control sample vs. $7.8 \%$ in the experimental sample) contributed to higher percentages of the microbiota in the experimental sample than in the control sample. The significant elevation of Bacteroidetes in the intestinal community of the starved seabass sample is in agreement with some other studies $[2,34,35]$ on dietary shifts. For example, in mice [34], fasting was associated with a significant increase in the proportional representation of the Bacteroidetes [from 20.6\% (fed) to $42.3 \%$ (fasted)]. Bacteroides with a much larger genome size (e.g., Bacteroides fragilis Strain NCTC9343: $5,205,140 \mathrm{bp})$ are normally mutualistic in the animal gastrointestinal flora. A large part of the proteins made by the Bacteroides genome are able to break down polysaccharides and metabolize their sugars [36]. They play a fundamental role in the processing of complex molecules to simpler ones in the host intestine. Their ability to harvest alternative energy sources from food might allow Bacteroides to be more competitive than other bacteria in the fish intestine during starvation. 
In contrast, Firmicutes are more abundant in the control sample (15.3\%) than in the experiment sample (10.1\%). The pattern that the relative abundance of Firmicutes in the gut is positively correlated with dietary caloric intake is frequently observed in humans, mice, pythons and in zebrafish [37,38]. In addition, the Betaproteobacteria class was more abundant with $20.8 \%$ in the control sample as compared with $7.9 \%$ in the experimental sample. The increased abundance of Betaproteobacteria class in response to feed was also observed in zebrafish [39], suggesting that Betaproteobacteria play a role in the interactions between microbiota, diet and hosts in fish.

To better understand the temporal changes of microbial community composition during starvation, we further amplified the 16S rRNA sequences from metagenomic DNA that was isolated directly from the intestine samples at three, six and twelve days post fast by using conserved primers targeting the domain Bacteria, and generated six libraries (one library per treatment per time) for Sanger sequencing. For each time point of the fasting challenges, around 350 high-quality sequences with a minimum length of $500 \mathrm{bp}$ were obtained. The taxonomic classification for the control library $\mathrm{d} 12$ (fed fishes) is drastically different from the control libraries $\mathrm{d} 3$ and $\mathrm{d} 6$ that were composed entirely of Cetobacterium. This is unexpected and may suggest that the classification is invalid at lower level (e.g., species and genus) due to a limitation of the BLAST-based approach. However, the phylogenetic analysis of the 16S rRNA gene data suggests a similar shift of bacteria components post the fasting challenge as our next generation sequencing-based metagenomic study (Additional file 2). For example, Bacteroidales were the most dominant order in the experimental samples, but were negligible in the control samples; Proteobacteria phylum (including Yersinia, Pectobacterium, Acinetobacter) and Fusobacteria phylum (including Cetobacterium) were the most dominant components in the control samples, but only low proportions of these bacteria were detected in the experimental samples at the three time points. Our data showed that the microbial community composition in the fish intestines can be quickly changed in response to starvation in less than three days. However, in human, a 10-day dietary intervention is not sufficient to alter the enterotype of an individual [40]. Compared to the changes of microbial composition in humans, the changes of the intestinal microbiota in fish are more rapid and significant. A recent comparison of the microbiota of zebrafish intestines and their housing water under fed and starved conditions suggested that some bacterial taxa observed in the fish intestine are found at similar frequency in the water, while other taxa are enriched specifically in the intestine [39]. This suggests that the fish gut looks a lot more like the surrounding environment, and even more so under starvation conditions due to a limited selective environment as compared to mammals.

\section{Metagenomic comparisons between fed and starved fishes by functional investigations}

Functional investigations by blasting the metagenomic data against public databases may reveal the genetic determinants of microbial interactions, and illuminate the mechanisms responsible for directing the changes in microbial diversity in response to fasting challenges. In order to generate a primary catalogue of microbial genes from the fish intestines, and explore the data differences caused from the fasting challenge, we first performed de novo assembly for the high quality reads. The de novo assembly of the high quality reads from two samples produced 326,789 scaffold sequences with a N50 length of 1,447 bp. Eighty-three percent of these reads was mapped back to the assembly. Of the scaffolding sequences, 299,018 sequences had a minimum length of $500 \mathrm{bp}$ and gave a total length of $443 \mathrm{Mb}$ with a N50 length of $1,857 \mathrm{bp}$. Sixty-one percent of the reads from the control sample and sixty-nine percent of the reads from the experimental sample were mapped back to the assembled sequences ( $\geq 500 \mathrm{bp}$ ). We then used the software Prodigal (Prokaryotic Dynamic Programming Gene finding Algorithm v2.15) [41] to predict the whole range of open reading frames (ORFs) for the metagenomic DNA sequences ( $\geq 500 \mathrm{bp}$ ). Even though a total of 462,828 ORFs were found, most of the ORFs appeared to be incomplete. We classified these predicted genes by aligning them to the Clusters of Orthologous Groups (COG) protein database that was derived from all of the proteins encoded by the genomes of bacteria, archaea and unicellular eukaryotes [42]. We found that 28,989 predicted ORFs were assigned to 3,182 COG orthologous groups, which could be classified into 30 COG functional categories (Additional files 3 and 4). Nearly six percent of the reads from the control sample and thirteen percent of the reads from the experimental sample were mapped back to the predicted ORF sequences with COG annotation.

There were significant differences in read counts of COG functional categories between the two datasets (Additional file 4). We found that three functional categories, including transcription, cell division and chromosome partitioning and replication, recombination and repair were significantly depleted in the experimental samples $(P<1 \mathrm{E}-15)$. Six functional categories, e.g., cell envelope biogenesis, outer membrane and defense mechanisms were significantly enriched during starvation $(P<1 \mathrm{E}-15$; Figure 2$)$.

To further identify genes in the intestinal microbiome, which are associated with starvation, statistical comparisons for the 3,182 COG orthologous groups were performed. Substantial fluctuations in the metagenomic components were found during starvation. We observed 


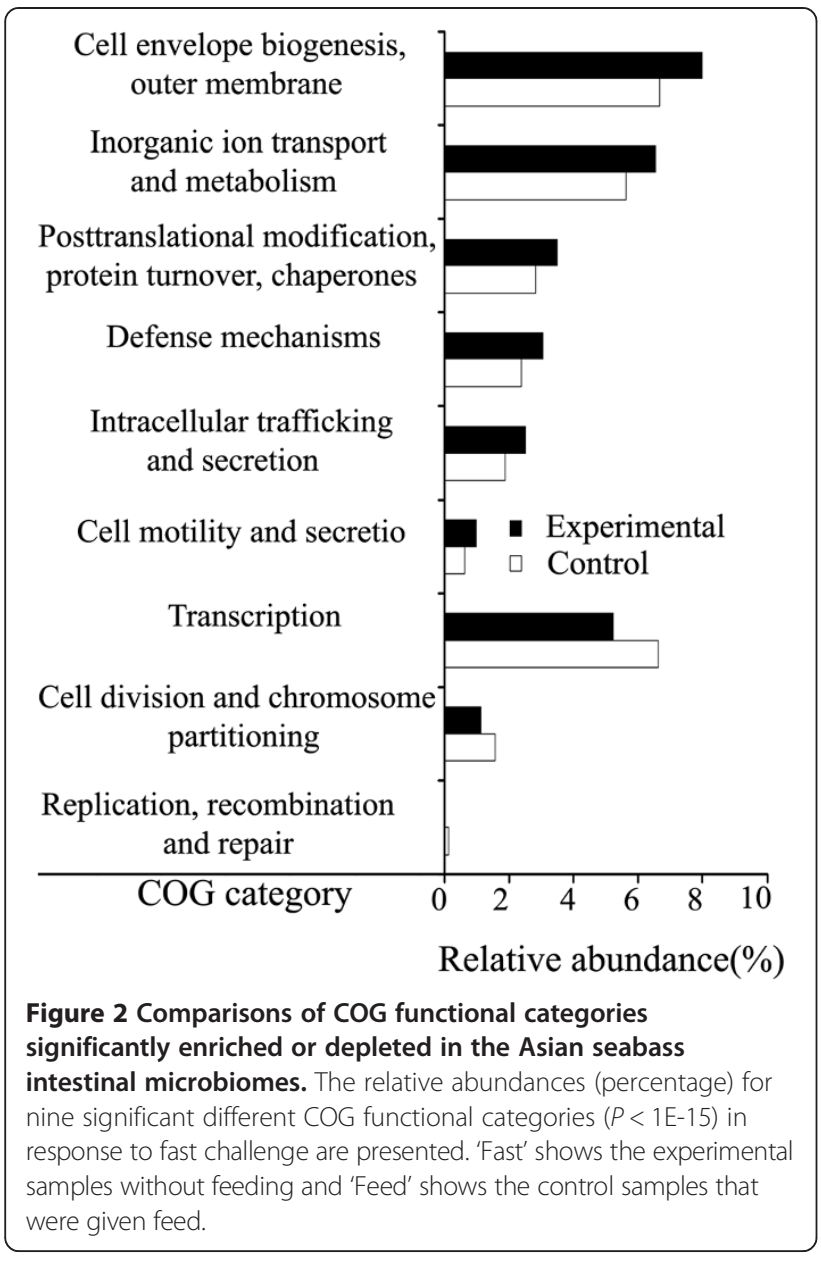

that 44 orthologous groups were significantly depleted (Additional file 5). These included the genes CitF (Citrate lyase, alpha subunit), EntF (Non-ribosomal peptide synthetase modules and related proteins) and transketolase (N-terminal subunit). These genes were mainly associated with energy production and conversion, secondary metabolites biosynthesis, transport, and catabolism, and carbohydrate transport and metabolism. Thirty genes were significantly enriched, including RhsA (Rhs family protein), NanH (Neuraminidase) and TesA (Lysophospholipase L1 and related esterases). Of the enriched genes, 16 (53\%) were associated with functional categories including Carbohydrate transport and metabolism, Inorganic ion transport and metabolism, and Amino acid transport and metabolism.

Gut microbiota provide their host with a physical barrier to pathogen infection by competitive exclusion and production of antimicrobial substances [43]. Antibiotics are produced by bacteria to outcompete intestinal pathogens. To further explore the microbe-microbe interactions, we downloaded 19,080 prokaryote genes related to antibiotic activity from UniProt protein database (http:// www.uniprot.org/). By using local blast alignment, 1,674 of the metagenomic sequences were mapped to 300 antibiotic related genes (Additional file 6). We found that seventy-two genes (e.g., $\mathrm{ABC}$ transporter related protein, dTDP-glucose 4,6-dehydratase, glucose-1-phosphate thymidylyltransferase, isoleucine-tRNA ligase 2) were significantly depleted in the experimental sample. Also seventy-two genes (e.g., efflux pump membrane transporter BepE, ribosomal RNA large subunit methyltransferase N) were significantly overrepresented in the experimental sample (Additional file 7). The overall mapping rate was only $1.67 \%$ in the control sample, while in the experimental sample, the rate was higher (9.42\%) (a ratio of 5.6 between two datasets). As compared with the overall mapping rate of the reads to COG orthologous groups (a ratio of 2 between the two datasets), a much higher proportion of reads in the experimental sample mapped to antibiotic related genes. Our data suggest that the relative proportions of bacteria with genes related to antibiotic activity may be increased in the fish intestine in response to starvation. Of the enriched genes, at least six genes were related to beta-lactamase activity, e.g., beta-lactamase type II, putative beta-lactamase hcpD, putative betalactamase hcpE and beta-lactamase blaTLA-1. An increase in antibiotic-producing strains could benefit the fish by excluding potential pathogens from colonizing the intestines. This observation could be due to the reduction in overall bacterial abundance with the exception of a few larger genome generalists (in Bacteroidetes) that harbor antibiotic resistance genes and can, in the conditions of nutrient deprivation, turn to harvesting host-produced products. This is in agreement to the human intestineadapted bacterial symbiont, which turns to host mucus glycans when polysaccharides are absent from the diet [44]. However, it is also possible that these genes may simply be hitching a ride with a microbe that is a generalist which can better survive these conditions.

\section{Functional interactions between intestinal microbiota and their hosts during starvation}

The importance of the intestinal microbiome in the development of both the intestinal mucosal and systemic immune systems have been shown in mammals [45]. In most species of fish, starvation is experienced during certain periods of every year largely due to environmental conditions [46,47]. Starvation affects many physiological changes to satisfy its energy requirements in fish [47]. Recent studies have shown that starvation can induce stress responses in fish. For example, starvation resulted in a significant reduction of the intestine length, the surface area of the intestinal mucosa and the mucosal thickness $[48,49]$ and increased xenobiotic resistance and paracellular permeability of epithelial cells in the anterior intestine [50]. To further explore the hostmicrobe interactions in fish intestine, ten genes related 
to immunity, defense and inflammatory response and four genes related to lipid metabolism and transport, and cholesterol metabolism were selected for expression analysis (Additional file 8). Temporal expression of the 14 genes was analyzed by qRT-PCR using RNA isolated from the Asian seabass intestine sampled at three, six and twelve days post fasting. The expression analysis clustered these genes into two groups (Figure 3). The apolipoprotein and phosphoethanolamine N-methyltransferase 3 genes related to lipid metabolism and transport, cholesterol metabolism $[51,52]$, and the placenta-specific gene 8 protein gene showing defense response to bacteria [53] were down-regulated in the starved experimental fish at all time-points of fasting (2.6 - 61.1 fold). Interestingly, mucin-2, which provides an insoluble mucous barrier that serves to protect the intestinal epithelium [54], was weakly increased at three days post fasting (1.1 fold), but quickly down-regulated at six and twelve days post fasting (2.2 2.3 fold). The intestinal barrier functions as one of the first lines of defense against microbial pathogens [55]. Dramatic decreases in the gene expression of mucin- 2 and placenta-specific gene 8 protein may lead to disruption of the mucosal barrier in the long term, increasing susceptibility to pathogen infections. Moreover, the immunerelated genes, such as complement $\mathrm{C} 1 \mathrm{q}$-like protein 2 (52-270 fold), class I histocompatibility antigen (20-22 fold) and CD46 (4-15 fold) were highly up-regulated from samples at three and six days post fast. However, at 12 days post fasting, immune related genes were only slightly increased, e.g., class I histocompatibility antigen ( 2 fold) and CD46 (2 fold), or down-regulated, e.g., complement C1qlike protein 2 (1.7 fold). These data suggest that long-term malnutrition or starvation will damage the mucosal barrier of the Asian seabass by increasing the permeability of the intestinal mucosal barrier. The intestinal microflora, especially the opportunistic pathogens could then cross the intestinal barrier when the intestinal mucosal barrier is damaged or the normal flora has been destroyed by antibiotics or nutrition deficiency. Based on prior findings from mammals, short-term stress experienced at the time of immune activation can enhance innate and adaptive immune responses, but long-term stress can suppress immunity by decreasing immune cell numbers and function and/or increasing active immunosuppressive mechanisms [56]. However, the underlying mechanisms of the nexus between host immune system and intestinal microbiota in response to nutrient changes need further exploration. Future studies targeting the microbial metaproteome and the interactions between the intestinal microbiota and their hosts in response to different nutrition conditions or stressors with a combination of continued sequencing, cultivation and functional genomics are likely to provide further insights into the functions of metagenomes in the fish intestine.

\section{Conclusions}

We determined the primary microbial gene catalogue and investigated the changes of the microbial composition and host gene expression in the intestine of Asian seabass in response to starvation. Proteobacteria (48.8\%), Firmicutes (15.3\%) and Bacteroidetes (8.2\%) were the three most abundant bacteria phyla. The components of intestinal microbiota community shifted in response to starvation with significant enrichment of Bacteroidetes,

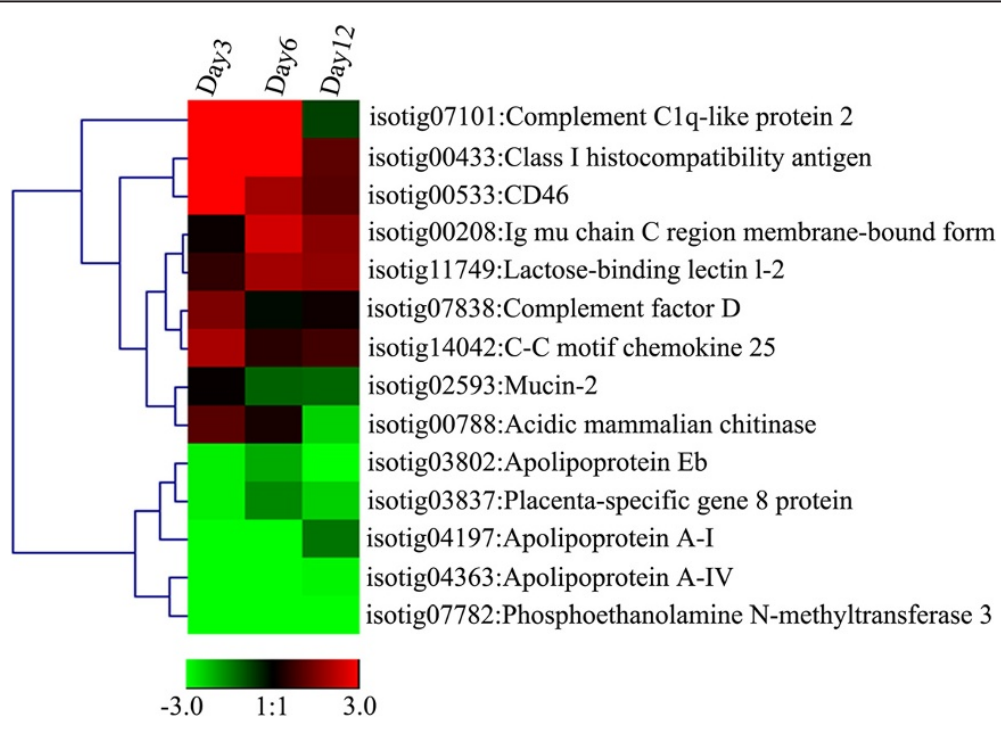

Figure 3 Gene expression profiles in the Asian seabass intestines across starvation treatments as revealed by qPCR. A heat map for fold changes of gene expression in 14 genes at three, six, twelve days post fast in the Asian seabass intestine is shown. Red indicates increased expression and green shows decreased expression in response to fast challenge. 
but depletion of Betaproteobacteria. There were significant differences in COG functional categories and orthologous groups, and genes related to antibiotic activity in response to starvation. Genes related to antibiotic activity in the microbiome were significantly enriched in response to starvation, and host genes related to immune system were generally up-regulated. This study provides the first insights into the intestinal microbiome and its changes under starvation in fish. Since our next generation sequencing datasets are from two pooled samples, the inter-individual variation can not be evaluated, which strongly influenced the power of our data analysis. Further detailed study on interactions between individual intestinal microbiomes and hosts under dynamic conditions will shed new light on how the hosts and microbes respond to the changing environment.

\section{Methods}

\section{Ethics statement}

All handling of fishes was conducted in accordance with the guidelines on the care and use of animals for scientific purposes set up by the Institutional Animal Care and Use Committee (IACUC) of the Temasek Life Sciences Laboratory, Singapore. The IACUC has specially approved this study within the project "Breeding of Asian seabass" (approval number is TLL (F)-12-004).

\section{Fish management, fasting challenge, sample collection and metagenomic DNA extraction}

An Asian seabass population was constructed by mass crossing of $50 \mathrm{~F} 1$ generation Asian seabass in the Marine Aquaculture Center, Singapore. The fingerlings were transferred to the animal facility of the Temasek Life Sciences Laboratory, Singapore. By gradually diminishing the salinity of the water, the fish was accustomed to living in freshwater. For challenge experiments, twelve fishes at the age of 11 months $(\sim 330 \mathrm{~g})$ were transferred equally to two 1,000 litre recirculating tanks with water volume of $~ 500$ litres in each tank. Nearly 30\% water was changed per day. Six fishes in the control tank were fed to satiation twice daily with pelleted feed (Biomar, Nersac, FR, France) that was kept in a cold room. The uneaten feed was removed from the bottom of the tank. The other six fishes in the test tank were not given access to feed before sampling. All fishes were euthanized by immersion in ice-water $\left(4^{\circ} \mathrm{C}\right.$ or less) for nearly $20 \mathrm{mi}$ nutes at 8 days post fasting. Deceased fish were then decontaminated with $70 \%$ ethanol and transferred into a laminar flow hood. Entire intestines were removed from each fish of each tank after dissection under sterile conditions. To isolate microbial cells, the pooled whole intestine from the fishes in the same tank were cut into small pieces and homogenized in $\sim 10$-fold dilution of cold sterile $1 \times$ phosphate-buffered saline solution using autoclaved mortars and pestles, which made it possible to isolate most of the gut wall-associated microbes. The mixtures were centrifuged at low speed, and the suspensions were filtered with a $100 \mu \mathrm{m}$ Nylon net filter (Millipore, Billerica, MA, USA). The filtrate was centrifuged at full speed for $20 \mathrm{~min}$. The cells were collected for isolation of metagenomic DNA using the QIAamp DNA Stool Mini Kit (Qiagen, Valencia, CA, USA) according to the manufacturer's instructions. DNA obtained was submitted to Macrogen Inc. (South Korea) for 100-cycle paired-end sequencing with the Illumina Hiseq 2000 sequencing system (Illumina, San Diego, CA, USA).

\section{Fasting and sampling of tissues for quantitative real-time PCR (qPCR) and 16S rRNA sequencing}

Thirty-six Asian seabass at the age of three months (body weight: $\sim 50 \mathrm{~g}$ ) originally maintained in a 1,000 L tank containing $500 \mathrm{~L}$ of freshwater were divided equally into two tanks containing 3,00 L of freshwater. Eighteen fishes, used as a control, were fed twice daily with pelleted feed (Biomar, Nersac, FR, France), and the other eighteen fishes in the test tank were not given access to feed before sampling. Six fishes from each group were sacrificed at three, six and twelve days post fasting, respectively. Before experiments, the pestles and dissecting tools were first soaked in 5\% concentration of Clorox bleach (The Clorox Company, Oakland, USA) for $\sim 15$ minutes, and autoclaved for 20 minutes after washing and rinsing in $\mathrm{ddH} 2 \mathrm{O}$. The working surface area and the deceased fish were decontaminated with $70 \%$ ethanol. Small sections $(\sim 1 \mathrm{~cm}$ of length) from the middle part of the intestine samples were taken and homogenized in $1 \mathrm{ml}$ of Trizol reagent (Invitrogen, Carlsbad, USA) with sterile KONTES $^{\circledR}$ pellet pestle driven by a cordless motor (Fisher Scientific, New Hampshire, USA). RNA isolation was conducted using the Trizol reagent (Invitrogen, Carlsbad, USA) according to the manufacturer's instructions. The remaining intestine samples from each fish was taken for DNA isolation using the QIAamp DNA Stool Mini Kit (Qiagen, Valencia, CA, USA) according to the manufacturer's instructions.

\section{S rRNA amplification, cloning and sequencing}

Bacterial 16S rRNAs were amplified by PCR using forward primer S-D-Bact-0008-a-S-20 (5' AGA GTT TGA TCC TGG CTC AG 3') [57], which targets the Bacteria domain, and the reverse primer $\mathrm{S}^{*}$-Univ-1492-b-A-21 (5' ACG GCT ACC TTG TTA CGA CTT 3') [58], which targets all living organisms. PCR was conducted as described in Suau et al. [59]. The purified products were ligated into the $\mathrm{pGEM}^{\ominus}-\mathrm{T}$ Vector Systems (Promega, Madison, USA) and then transformed into E. coli strain XL-1 (Stratagene, CA, USA). Randomly picked clones from the libraries were sequenced by using single pass 
sequencing using M13 forward sequencing primer (Promega, Madison, USA) with a 3730 xl DNA analyzer (Applied Biosystems, Foster city, CA).

\section{Analysis of seabass intestinal gene expression with qPCR} From published transcriptome datasets of the Asian seabass [60-62], 14 genes related to immune and metabolism activity were selected for analysis of gene expressions in seabass intestines by qPCR (Additional file 8). One primer pair for each gene was designed using the program PrimerSelect (DNASTAR, Wilmington, DE, USA). Gene expression was analyzed as described in Xia et al. [61]. Briefly, equal aliquots of total RNA from each of the six fish intestines under the same conditions were pooled, and around $1 \mu \mathrm{g}$ of the DNase I-treated total RNA were reverse transcribed to cDNA by MMLV reverse transcriptase (Promega, Madison, Wisconsin, USA) with random hexamer primers. PCR was performed in triplicate with the iQ SYBR Green Master Mix as described by the manufacturer in an $\mathrm{iQ}^{\mathrm{Tm}} 5$ Real Time PCR Detection System (Bio-Rad, Hercules, CA, USA). Ten-fold dilutions of the CDNA preparation were used as DNA templates. For the PCR reaction in a total volume of $20 \mu \mathrm{l}$, a master reaction mix (per tube) contains $10 \mu \mathrm{l}$ of Supermix, $0.3 \mu \mathrm{l}$ of forward primer (300 $\mathrm{nM}$ final), $0.3 \mu \mathrm{l}$ of reverse primer (300 nM final), and $1 \mu \mathrm{l}$ of the diluted cDNA template. Thermal cycling conditions were as follows: $3 \mathrm{~min}$ at $95^{\circ} \mathrm{C}$, followed by 40 cycles: $95^{\circ} \mathrm{C}$ for $15 \mathrm{sec}$, annealing temperature for $30 \mathrm{sec}, 72^{\circ} \mathrm{C}$ for $30 \mathrm{sec}$. The PCR products for each of the primer pairs giving one specific band were confirmed on $2 \%$ agarose gels stained with Ethidium Bromide fluorescence under ultraviolet light and by melting curve analysis (only one peak was observed). Elongation factor-1 alpha gene (EF1A) has been suggested as the reference gene in qRT-PCR assays $[63,64]$. For analysis of the change of gene expression, the values of triplicate qPCR reactions were normalized to EF1A gene expression, calculated by the $\Delta \Delta C T$ method. The normalized expression values were then used to construct a heat map by Cluster 3.0 with parameter settings as hierarchical clustering, uncentered correlation and complete linkage (http://bonsai.ims.u-tokyo. ac.jp/ mdehoon/software/cluster/software.htm\#ctv).

\section{De novo assembly of the intestine metagenomes for the Asian seabass}

Paired-end data were processed to filter the low quality reads and adaptors using the CLC Genomics Workbench (CLC bio, Cambridge, MA). De novo assembly of the high-quality short reads was carried out using the SOAPdenovo Assembler (V1.05) [65] with assembly parameters '-d -D - R - F -K 53'. After assembly, the resulting genomic sequences with a length less than 500 bp were filtered out with software NGS QC Toolkit [66]. The software packages Bowtie 2 [67] and SAMtools [68] were applied for aligning sequencing reads to the assembled genomic sequences and retrieving statistical data.

\section{Phylogenetic analysis, gene prediction and functional classification of the metagenomic data}

MetaPhlAn (v1.7.3) is a computational tool for profiling the composition of microbial communities from metagenomic shotgun sequencing data by running BLAST searches against unique clade-specific marker genes identified from 3,000 reference genomes [29]. This tool was used for profiling the composition of microbial communities from our sequencing reads. The assembled genomic sequences were used to predict the whole range of ORFs with Prodigal (Prokaryotic Dynamic Programming Genefinding Algorithm v2.15) [41]. The predicted ORFs were then aligned to the protein database of Clusters of Orthologous Groups (COG) database [69] using BLASTp algorithm with an E-value threshold of E-7. Prokaryote protein gene sequences with the biological process category annotated as antibiotic resistance or antibiotic biosynthesis were downloaded from UniProt protein databases and used for gene functional analysis.

Taxonomic assignment for high quality $16 \mathrm{~S}$ rRNA sequences was carried out using the Ribosomal Database Project (RDP; http://rdp.cme.msu.edu/) [70]. The rRNA analysis files downloaded from the RDP website were then imported into the software MEGAN [71] to analyze the taxonomic content of DNA reads.

\section{Identification of changed genes, pathways and taxonomic} units in fed vs. starved gut microbiomes

The online tool Xipe (written by Beltran Rodriguez-Muller; http://edwards.sdsu.edu/cgi-bin/xipe.cgi) provides a nonparametric statistical analysis of the distribution of samples. This tool can be used to compare two different populations and identify the differences between those samples. The STAMP (Statistical Analysis of Metagenomic Profiles) [72] is a software package for analyzing metagenomic profiles. Both tools have been applied or suggested in previous metagenomic studies [73,74]. In our data analysis, the proportions of the relative abundance profiles of the composition of microbial communities exported from the software MetaPhlAn were first adjusted to read counts per million mapped. Bootstrap analyses of enrichment and depletion of bacterial taxa, genes and pathways between dietary treatments were carried out using the tool Xipe (with the parameters: sample size of 5,000 and confidence level of 0.9) and the software STAMP (with the following parameters: Bootstrap as statistical test, asymptotic as confidence interval method, Storey's FDR as multiple test correction method and 10,000 bootstrap replicates). A feature is 
considered significantly different if it shows significant differences in both analyses.

\section{Availability of supporting data}

The 16S rRNA sequences have been deposited in NCBI under accession \#s: KC599554 - KC601631. The metagenomic sequence data are available under the DNA Data Bank of Japan (DDBJ) Sequence Read Archive database (accession no.: [DDBJ: DRR004449 and DRR004450]).

\section{Additional files}

Additional file 1: Taxonomic profiles detected in the intestinal metagenomes of the Asian seabass.

Additional file 2: Comparison of the intestinal bacteria variation in Asian seabass as detected by $16 \mathrm{~S}$ rRNA sequencing in response to starvation. The generated $16 \mathrm{~S}$ rRNA sequences for each sample are normalized to the total number of the sequences. Each unique color represents a sample. Each circle represents one taxon.

The area size for each color within a circle is proportional to the relative abundance of one taxon in different samples. The samples at three, six, twelve days post fast are named as Fast-d3, $-\mathrm{d} 6$ and $-\mathrm{d} 12$, and the controls are named as Feed-d3, $-\mathrm{d} 6$ and $-\mathrm{d} 12$, respectively.

Additional file 3: The information of significant annotation of the intestine metagenomes of the Asian seabass against COG database.

Additional file 4: Comparison of COG functional categories of the intestinal metagenomes in the Asian seabass in response to starvation.

Additional file 5: COG orthologus groups significantly enriched or depleted in the intestinal microbiome of the Asian seabass.

Additional file 6: Genes in the Asian seabass intestinal microbiome involved in antibiotic activity.

Additional file 7: Genes with antibiotic activity significantly enriched or depleted in the intestinal microbiome.

Additional file 8: Primers used in the expression analysis of selected genes from the Asian seabass.

\section{Abbreviations}

COG: Clusters of orthologous groups; rRNA: Ribosomal RNA; NGS: Nextgeneration sequencing; ORF: Open reading frame; qPCR: Quantitative real-time PCR; DDBJ: DNA Data Bank of Japan.

\section{Competing interests}

The authors declare that they have no competing interests.

\section{Authors' contributions}

JHX performed the metagenomic data analysis. GL optimized the protocol for isolation of metagenomic DNA and extracted the DNA. FGH and XJL prepared the RNA samples, and WZY and ML performed gene expression by qRT-PCR. WL managed the fish. JHX and GHY conceived of and supervised the study. JHX wrote the manuscript. YGH finalized the manuscript. All authors read and approved the final manuscript.

\section{Acknowledgements}

This research is supported by the National Research Foundation, Prime Minister's Office, Singapore under its Competitive Research Program (CRP Award No. NRF-CRP002-001). We thank MAC, AVA for long-time co-operation on Asian seabass breeding and our lab members for assisting in tissue sample collection.

Received: 27 July 2013 Accepted: 31 March 2014

Published: 5 April 2014

\section{References}

1. Okada T, Fukuda S, Hase K, Nishiumi S, Izumi Y, Yoshida M, Hagiwara T, Kawashima R, Yamazaki M, Oshio T, Otsubo T, Inagaki-Ohara K, Kakimoto K, Higuchi K, Kawamura Yl, Ohno H, Dohi T: Microbiota-derived lactate accelerates colon epithelial cell turnover in starvation-refed mice. Nat Commun 2013, 4:1654.

2. Furet JP, Kong LC, Tap J, Poitou C, Basdevant A, Bouillot JL, Mariat D, Corthier G, Doré J, Henegar C, Rizkalla S, Clément K: Differential adaptation of human gut microbiota to bariatric surgery-induced weight loss: links with metabolic and low-grade inflammation markers. Diabetes 2010, 59(12):3049-3057.

3. Goodlad RA, Wright NA: The effects of starvation and refeeding on intestinal cell proliferation in the mouse. Virchows Arch B Cell Pathol Ind Mol Pathol 1984, 45(1):63-73.

4. Ley RE, Turnbaugh PJ, Klein S, Gordon Jl: Microbial ecology: human gut microbes associated with obesity. Nature 2006, 444(7122):1022-1023.

5. Tremaroli $V$, Backhed F: Functional interactions between the gut microbiota and host metabolism. Nature 2012, 489(7415):242-249.

6. Moore AM, Munck C, Sommer MO, Dantas G: Functional metagenomic investigations of the human intestinal microbiota. Front Microbio/ 2011, 2:188.

7. Kelly P: Symposium 4: Gut function: effects on over- and undernutrition nutrition, intestinal defence and the microbiome. Proc Nutr Soc 2010, 69(2):261-268.

8. O'Mahony SM, Marchesi JR, Scully P, Codling C, Ceolho AM, Quigley EM, Cryan JF, Dinan TG: Early life stress alters behavior, immunity, and microbiota in rats: implications for irritable bowel syndrome and psychiatric illnesses. Biol Psychiatry 2009, 65(3):263-267.

9. Tanaka S, Kobayashi T, Songjinda P, Tateyama A, Tsubouchi M, Kiyohara C, Shirakawa T, Sonomoto K, Nakayama J: Influence of antibiotic exposure in the early postnatal period on the development of intestinal microbiota. Fems Immunol Med Mic 2009, 56(1):80-87.

10. Turnbaugh PJ, Hamady M, Yatsunenko T, Cantarel BL, Duncan A, Ley RE, Sogin ML, Jones WJ, Roe BA, Affourtit JP, Egholm M, Henrissat B, Heath AC, Knight R, Gordon Jl: A core gut microbiome in obese and lean twins. Nature 2009, 457(7228):480-484.

11. Hopkins MJ, Sharp R, Macfarlane GT: Variation in human intestinal microbiota with age. Digest Liver Dis 2002, 34:S12-S18.

12. Schmidt B, Mulder IE, Musk CC, Aminov RI, Lewis M, Stokes CR, Bailey M, Prosser JI, Gill BP, Pluske JR, Kelly D: Establishment of normal gut microbiota is compromised under excessive hygiene conditions. Plos One 2011, 6(12):e28284.

13. Dethlefsen L, Eckburg PB, Bik EM, Relman DA: Assembly of the human intestinal microbiota. Trends Ecol Evol 2006, 21(9):517-523.

14. Brown K, DeCoffe D, Molcan E, Gibson DL: Diet-induced dysbiosis of the intestinal microbiota and the effects on immunity and disease. Nutrients 2012, 4(11):1552-1553.

15. Morgan XC, Tickle TL, Sokol H, Gevers D, Devaney KL, Ward DV, Reyes JA, Shah SA, LeLeiko N, Snapper SB, Bousvaros A, Korzenik J, Sands BE, Xavier RJ, Huttenhower C: Dysfunction of the intestinal microbiome in inflammatory bowel disease and treatment. Genome Biol 2012, 13(9):R79.

16. Caporaso JG, Lauber CL, Costello EK, Berg-Lyons D, Gonzalez A, Stombaugh J, Knights D, Gajer P, Ravel J, Fierer N, Gordon Jl, Knight R: Moving pictures of the human microbiome. Genome Biol 2011, 12(5):R50.

17. Kuczynski J, Costello EK, Nemergut DR, Zaneveld J, Lauber CL, Knights D, Koren O, Fierer N, Kelley ST, Ley RE, Gordon Jl, Knight R: Direct sequencing of the human microbiome readily reveals community differences. Genome Biol 2010, 11(5):210.

18. Ponten TS: Metatranscriptomics of the human gut microbiome. Genome Biol 2011, 12:3-3.

19. Segata N, Haake SK, Mannon P, Lemon KP, Waldron L, Gevers D, Huttenhower C, Izard J: Composition of the adult digestive tract bacterial microbiome based on seven mouth surfaces, tonsils, throat and stool samples. Genome Biol 2012, 13(6):R42.

20. Weinstock GM: The volatile microbiome. Genome Biol 2011, 12(5):114

21. Zeeuwen $P$, Boekhorst J, van den Bogaard EH, de Koning HD, van de Kerkhof PMC, Saulnier DM, van Swam II, van Hijum S, Kleerebezem M, Schalkwijk J, Timmerman HM: Microbiome dynamics of human epidermis following skin barrier disruption. Genome Biol 2012, 13(11):R101 
22. Vaishampayan PA, Kuehl JV, Froula $J \mathrm{~L}$, Morgan $\mathrm{J}$, Ochman $\mathrm{H}$, Francino MP: Comparative metagenomics and population dynamics of the gut microbiota in mother and infant. Genome Biol Evol 2010, 2:53-66.

23. Thomas T, Gilbert J, Meyer F: Metagenomics - a guide from sampling to data analysis. Microb Inform Exp 2012, 2(1):3

24. Han SF, Liu YC, Zhou ZG, He SX, Cao YA, Shi PJ, Yao B, Ringo E: Analysis of bacterial diversity in the intestine of grass carp (Ctenopharyngodon idellus) based on 16S rDNA gene sequences. Aquac Res 2010, 42(1):47-56.

25. Wu S, Wang G, Angert ER, Wang W, Li W, Zou H: Composition, diversity, and origin of the bacterial community in grass carp intestine. Plos One 2012, 7(2):e30440.

26. Roeselers G, Mittge EK, Stephens WZ, Parichy DM, Cavanaugh CM, Guillemin K, Rawls JF: Evidence for a core gut microbiota in the zebrafish. ISME 2011, 5(10):1595-1608

27. Larson H: Order Perciformes. Suborder Percoidei. Centropomidae. Sea perches. In FAO species identification guide for fishery purposes The living marine resources of the Western Central Pacific, Volume 4. Edited by Carpenter KE, Niem VH. Rome: FAO; 1999:2429-2432.

28. Gilbert JA, Dupont CL: Microbial metagenomics: beyond the genome. Annu Rev Mar Sci 2011, 3:347-371.

29. Segata N, Waldron L, Ballarini A, Narasimhan V, Jousson O, Huttenhower C Metagenomic microbial community profiling using unique clade-specific marker genes. Nat Methods 2012, 9(8):811.

30. Pop M: We are what we eat: how the diet of infants affects their gut microbiome. Genome Biol 2012, 13(4):152.

31. Schwartz S, Friedberg I, Ivanov IV, Davidson LA, Goldsby JS, Dahl DB, Herman D, Wang M, Donovan SM, Chapkin RS: A metagenomic study of diet-dependent interaction between gut microbiota and host in infants reveals differences in immune response. Genome Biol 2012, 13(4):R32

32. Qin J, Li R, Raes J, Arumugam M, Burgdorf KS, Manichanh C, Nielsen T, Pons N, Levenez F, Yamada T, Mende DR, Li J, Xu J, Li S, Li D, Cao J, Wang B, Liang $H$, Zheng $H$, Xie $Y$, Tap J, Lepage P, Bertalan M, Batto JM, Hansen T, Le Paslier D, Linneberg A, Nielsen HB, Pelletier E, Renault P: A human gut microbial gene catalogue established by metagenomic sequencing. Nature 2010, 464(7285):59-65.

33. Ley RE, Lozupone CA, Hamady M, Knight R, Gordon Jl: Worlds within worlds: evolution of the vertebrate gut microbiota. Nat Rev Microbiol 2008, 6(10):776-788

34. Crawford PA, Crowley JR, Sambandam N, Muegge BD, Costello EK, Hamady M, Knight R, Gordon Jl: Regulation of myocardial ketone body metabolism by the gut microbiota during nutrient deprivation. Proc Natl Acad Sci U S A 2009, 106(27):11276-11281.

35. Murphy EF, Cotter PD, Healy S, Marques TM, O'Sullivan O, Fouhy F, Clarke SF, O'Toole PW, Quigley EM, Stanton C, Ross PR, O'Doherty RM, Shanahan F: Composition and energy harvesting capacity of the gut microbiota: relationship to diet, obesity and time in mouse models. Gut 2010, 59:1635-1642.

36. Xu J, Bjursell MK, Himrod J, Deng S, Carmichael LK, Chiang HC, Hooper LV, Gordon Jl: A genomic view of the human-Bacteroides thetaiotaomicron symbiosis. Science 2003, 299(5615):2074-2076.

37. Salyers AA, Gupta A, Wang YP: Human intestinal bacteria as reservoirs for antibiotic resistance genes. Trends Microbiol 2004, 12(9):412-416.

38. Lofmark S, Jernberg C, Jansson JK, Edlund C: Clindamycin-induced enrichment and long-term persistence of resistant Bacteroides spp. and resistance genes. J Antimicrob Chemoth 2006, 58(6):1160-1167.

39. Semova I, Carten JD, Stombaugh J, Mackey LC, Knight R, Farber SA, Rawls JF: Microbiota regulate intestinal absorption and metabolism of fatty acids in the zebrafish. Cell host microbe 2012, 12(3):277-288.

40. Wu GD, Chen J, Hoffmann C, Bittinger K, Chen YY, Keilbaugh SA, Bewtra M Knights D, Walters WA, Knight R, Sinha R, Gilroy E, Gupta K, Baldassano R, Nessel L, Li HZ, Bushman FD, Lewis JD: Linking long-term dietary patterns with gut microbial enterotypes. Science 2011, 333(6052):105-108.

41. Hyatt D, Chen GL, LoCascio PF, Land ML, Larimer FW, Hauser LJ: Prodigal: prokaryotic gene recognition and translation initiation site identification. BMC Bioinforma 2010, 11:119.

42. Koonin EV: The Clusters of Orthologous Groups (COGs) Database: phylogenetic classification of proteins from complete genomes. In The NCBI Handbook. Edited by McEntyre J, Ostell J. Bethesda (MD): National Center for Biotechnology Information (US); 2002
43. Sekirov I, Russell SL, Antunes LC, Finlay BB: Gut microbiota in health and disease. Physiol Rev 2010, 90(3):859-904

44. Sonnenburg JL, Xu J, Leip DD, Chen CH, Westover BP, Weatherford J, Buhler JD, Gordon Jl: Glycan foraging in vivo by an intestine-adapted bacterial symbiont. Science 2005, 307(5717):1955-1959.

45. Macpherson AJ, Harris NL: Interactions between commensal intestinal bacteria and the immune system. Nat Rev Immunol 2004, 4(6):478-485.

46. Tripathi G, Verma P: Starvation-induced impairment of metabolism in a freshwater catfish. Z Naturforsch C 2003, 58(5-6):446-451.

47. Johnston IA: Quantitative analysis of muscle breakdown during starvation in the marine flatfish Pleuronectes platessa. Cell Tissue Res 1981, 214(2):369-386.

48. Montgomery $\mathrm{WL}$, Pollak PE: Gut anatomy and $\mathrm{pH}$ in a red sea surgeonfish, canthurus nigrofuscus. Mar Ecol Prog Ser 1988, 44:7-13.

49. Hall KC, Bellwood DR: Histological effects of cyanide, stress and starvation on the intestinal mucosa of Pomacentrus coelestis, a marine aquarium fish species. J Fish Biol 1995, 47(3):438-454

50. Baumgarner BL, Bharadwaj AS, Inerowicz D, Goodman AS, Brown PB: Proteomic analysis of rainbow trout (Oncorhynchus mykiss) intestinal epithelia: physiological acclimation to short-term starvation. Comp Biochem Physiol Part D Genomics Proteomics 2013, 8(1):58-64.

51. Cham BE: Importance of apolipoproteins in lipid metabolism. Chem Biol Interact 1978, 20(3):263-277.

52. Jost R, Berkowitz O, Shaw J, Masle J: Biochemical characterization of two wheat phosphoethanolamine $\mathrm{N}$-methyltransferase isoforms with different sensitivities to inhibition by phosphatidic acid. J Biol Chem 2009, 284:31962-31971.

53. Zheng W, Liu G, Ao J, Chen X: Expression analysis of immune-relevant genes in the spleen of large yellow croaker (Pseudosciaena crocea) stimulated with poly I:C. Fish Shellfish Immunol 2006, 21(4):414-430.

54. Nungester WJ, Wolf AA, Jourdonais LF: Effect of gastric mucin on virulence of bacteria in intraperitoneal injections in the mouse. Proc Soc Exp Biol Med 1932, 30(2):120-121

55. Blum S, Schiffrin EJ: Intestinal microflora and homeostasis of the mucosa immune response: implications for probiotic bacteria? Curr Issues Intest Microbiol 2003, 4(2):53-60.

56. Dhabhar FS: Enhancing versus suppressive effects of stress on immune function: implications for immunoprotection and immunopathology. Neuroimmunomodulation 2009, 16(5):300-317.

57. Hicks RE, Amann RI, Stahl DA: Dual staining of natural bacterioplankton with 4',6-Diamidino-2-Phenylindole and fluorescent oligonucleotide probes targeting kingdom-level 16 s Ribosomal-Rna sequences. Appl Environ Microb 1992, 58(7):2158-2163.

58. Kane MD, Poulsen LK, Stahl DA: Monitoring the enrichment and isolation of Sulfate-reducing bacteria by using oligonucleotide hybridization probes designed from environmentally derived $16 \mathrm{~s}$ ribosomal-Rna sequences. Appl Environ Microb 1993, 59(3):682-686.

59. Suau A, Bonnet R, Sutren M, Godon JJ, Gibson GR, Collins MD, Dore J: Direct analysis of genes encoding 16S rRNA from complex communities reveals many novel molecular species within the human gut. Appl Environ Microb 1999, 65(11):4799-4807.

60. Xia $\mathrm{JH}$, Yue $\mathrm{GH}$ : Identification and analysis of immune-related transcriptome in Asian seabass Lates calcarifer. BMC Genomics 2010, 11:356.

61. Xia JH, He XP, Bai ZY, Lin G, Yue GH: Analysis of the Asian Seabass Transcriptome Based on Expressed Sequence Tags. DNA Res 2011 18(6):513-522

62. Xia JH, Liu P, Liu F, Lin G, Sun F, Tu RJ, Yue GH: Analysis of stressresponsive transcriptome in the intestine of Asian seabass (Lates calcarifer) using RNA-Seq. DNA Res 2013, 20(5):449-460.

63. Peña AA, Bols NC, Marshall SH: An evaluation of potential reference genes for stability of expression in two salmonid cell lines after infection with either Piscirickettsia salmonis or IPNV. BMC Res Notes 2010, 3:101.

64. Leelatanawit R, Klanchui A, Uawisetwathana U, Karoonuthaisiri N: Validation of reference genes for real-time PCR of reproductive system in the black tiger shrimp. Plos One 2012, 7(12):e52677.

65. Li RQ, Zhu HM, Ruan J, Qian WB, Fang XD, Shi ZB, Li YR, Li ST, Shan G, Kristiansen K, Li SG, Yang HM, Wang J, Wang J: De novo assembly of human genomes with massively parallel short read sequencing Genome Res 2010, 20(2):265-272.

66. Patel RK, Jain M: NGS QC Toolkit: a toolkit for quality control of next generation sequencing data. Plos One 2012, 7(2):e30619. 
67. Langmead B, Salzberg SL: Fast gapped-read alignment with Bowtie 2 . Nat Methods 2012, 9(4):357-359.

68. Li H, Handsaker B, Wysoker A, Fennell T, Ruan J, Homer N, Marth G, Abecasis G, Durbin R: The sequence alignment/map format and SAMtools. Bioinformatics 2009, 25(16):2078-2079.

69. Tatusov RL, Fedorova ND, Jackson JD, Jacobs AR, Kiryutin B, Koonin EV Krylov DM, Mazumder R, Mekhedov SL, Nikolskaya AN, Rao BS, Smirnov S, Sverdlov AV, Vasudevan S, Wolf YI, Yin JJ, Natale DA: The COG database: an updated version includes eukaryotes. BMC Bioinforma 2003, 4:41.

70. Wang Q, Garrity GM, Tiedje JM, Cole JR: Naive Bayesian classifier for rapid assignment of rRNA sequences into the new bacterial taxonomy. Appl Environ Microb 2007, 73(16):5261-5267.

71. Huson DH, Auch AF, Qi J, Schuster SC: MEGAN analysis of metagenomic data. Genome Res 2007, 17(3):377-386.

72. Parks $\mathrm{DH}$, Beiko RG: Identifying biologically relevant differences between metagenomic communities. Bioinformatics 2010, 26(6):715-721.

73. Logares R, Haverkamp THA, Kumar S, Lanzénd A, Nederbragtc AJ, Quinceg C, Kauserud H: Environmental microbiology through the lens of highthroughput DNA sequencing: Synopsis of current platforms and bioinformatics approaches. J Microbiol Methods 2012, 91:106-113.

74. Dinsdale EA, Pantos O, Smriga S, Edwards RA, Angly F, Wegley L, Hatay M, Hall D, Brown E, Haynes M, Krause L, Sala E, Sandin SA: Microbial ecology of four coral atolls in the northern line islands. Plos One 2008, 3(2):e1584

doi:10.1186/1471-2164-15-266

Cite this article as: Xia et al:: The intestinal microbiome of fish under starvation. BMC Genomics 2014 15:266.

\section{Submit your next manuscript to BioMed Central and take full advantage of:}

- Convenient online submission

- Thorough peer review

- No space constraints or color figure charges

- Immediate publication on acceptance

- Inclusion in PubMed, CAS, Scopus and Google Scholar

- Research which is freely available for redistribution 